\title{
POINTWISE SMOOTHNESS, TWO-MICROLOCALIZATION AND WAVELET COEFFICIENTS
}

\author{
S. JAFFARD
}

In this paper, we shall compare three notions of pointwise smoothness: the usual definition, I.M. Bony's two-microlocal spaces $C_{x_{0}}^{s, s^{\prime}}$, and the corresponding definition on the wavelet coefficients. The purpose is mainly to show that these two-microlocal spaces provide "good substitutes" for the pointwise Hölder regularity condition; they can be very precisely compared with this condition, they have more functional properties, and can be characterized by conditions on the wavelet coefficients. We also give applications of these properties. In Part 2 some results on the microlocal spaces contained in [B2] will be recalled. Theorems 3 and 4 are also essentially contained in [B2]. The starting point of this paper was a note ([J1]) the author had written on a comparison between the Holder criterion of regularity at a given point $x_{0}$ and a corresponding property defined on the wavelet coefficients. Some easy proofs are omitted or abridged and can be found in [J2].

\section{Pointwise smoothness and two-microlocalization}

Let $s$ be a strictly positive real number. Let us recall the usual definition of the Hölder criterion at $x_{0}$. A function $f$ betongs to $C_{x_{0}}^{*}$ if there exists a polynomial $P(x)$ of degree equal to the integral part of $s$ such that

$$
f(x)=P(x)+O\left(\left|x-x_{0}\right|^{s}\right) .
$$

The following properties are classical. If $f$ belongs to $C_{x_{0}}^{s}$, nothing is implied on the derivatives of $f$. In dimension 1 , the primitive of $f$ belongs to $C_{x_{0}}^{*+1}$. In dimension larger than $I$, the fractional integration of order 1 maps $C_{x_{0}}^{s}$ into $C_{x_{0}+1}^{s+1}$

The two-microlocalization of J.M. Bony consists in replacing the preceding notion by another one which allows to derivate and integrate. The classical pseudo-differential operators will operate on these spaces, which will be spaces of temperate distributions.

These spaces are defined by conditions on the Littlewood-Paley decomposition. Let us recall its definition. Let $\theta$ be a function in the Schwartz class such that

$$
\theta(\xi)=1 \text { if }|\xi| \leq 1 / 2 \text { and } \theta(\xi)=0 \text { if }|\xi| \geq 1
$$


Let then

$$
\eta(\xi)=\theta(\xi / 2)-\theta(\xi)
$$

Let $S_{j}$ be the "low-pass filter", which, after a Fourier transform, is a multiplication by $\theta\left(2^{-j} \xi\right)$. Define $\Delta_{j}=S_{j+1}-S_{j}$. Thus

$$
I=S_{0}+\Delta_{0}+\Delta_{\mathrm{I}}+\ldots
$$

The Fourier transform of $\Delta_{j}(u)$ is supported by the set

$$
2^{j-1} \leq|\xi| \leq 2^{j+1} \text {. }
$$

The two-microlocal spaces can now be defined.

Definition 1. Let $s$ and $s^{\prime}$ be two real numbers; $C_{0}^{8, v^{\prime}}$ is the Banach space of distributions such that

$$
\left|S_{0}(u)(x)\right| \leq C(1+|x|)^{-s^{\prime}}
$$

and

$$
|\Delta j(u)(x)| \leq C 2^{-j s}\left(1+\left|2^{j} x\right|\right)^{-s^{\prime}} .
$$

The space $C_{x_{0}}^{s, s^{\prime}}$ is then obtained through a simple translation. If $s^{\prime}=0$, the space thus obtained is the global Hölder space $C^{s}\left(\mathbf{R}^{n}\right)$. The effect of $s^{t}$ is to accentuate either the role played by $x_{0}$, when $s^{t}<0$, or the behavior at infinity, when $s^{\prime}>0$. A few other remarks will give a better understanding of these conditions.

Define $u_{j}=\Delta_{j}(u)$ and $U_{j}(x)=u_{j}\left(2^{-j} x\right)$. Then, the Fourier transform of $U_{j}$ is a distribution carried by the set $1 / 2 \leq|\xi| \leq 2$, and (3) implies that

$$
\left|U_{j}(u)(x)\right| \leq C 2^{-j s}(1+|x|)^{-s^{\prime}}
$$

Such an estimate is stable under derivation and fractional integration, more generally under the action of the operators $(I-\Delta)^{s / 2}$ or $(-\Delta)^{y / 2}, s \in \mathbf{R}$, because these operators are Fourier multipliers which, once restricted to $1 / 2 \leq$ $|\xi| \leq 2$, coincide with a function of the Schwartz class. Coming back to the "space variable", we get

$$
(-\Delta)^{s / 2} U_{j}=K_{s} * U_{j}
$$

where $K_{s}$ belongs to the Schwartz class.

This convolution operator preserves the polynomial increase or decay, as it appears in (4). So that the following equivalence holds

$$
u \in C_{x_{0}}^{s, s^{\prime}} \Longleftrightarrow \frac{\partial u}{\partial x_{j}} \in C_{x_{0}}^{s-1, s^{\prime}} \text { for } 1 \leq j \leq n
$$

In the following part, we shall investigate the nature of the elements of $C_{z_{0}}^{s, g^{\prime}}$, whether they are (eventually smooth) functions or distributions. 


\section{The elements of $C_{x_{0}}^{s, s^{\prime}}$}

We claim that the elements of $C_{x_{0}}^{s,-s}$ are (in general) distributions. To shorten the proof, suppose $n=1, x_{0}=0$ and $0<s<1$. Define

$$
\theta(x)=\phi *|x|^{3},
$$

where the Fourier transform of $\phi$ belongs to the Schwartz class, vanishes outside $[-1 / 2,1 / 2]$, and is equal to 1 on $[-1 / 4,1 / 4]$. So that, for any $N \geq 1$,

$$
\theta(x)=|x|^{s}+O\left(|x|^{-N}\right) \text {. }
$$

Define then

$$
f(x)=\sum_{3}^{\infty} 2^{-j s} \theta\left(2^{j} x\right) e^{i 2^{j} x}=\sum_{0}^{\infty} u_{j}(x)
$$

Then

$$
u_{j}(x)=2^{-j s} u\left(2^{j} x\right) \text { and }|u(x)| \leq C(1+|x|)^{s},
$$

so that $f$ belongs to $C_{0}^{s_{r}-s}$. The restriction of $f$ to any interval $] \delta / 2, \delta[, \delta>0$, is a distribution, because, if $\delta / 2<x<\delta$,

$$
f(x)=|x|^{s} \sum_{0}^{\infty} e^{i 2^{\prime} x}+O(1)
$$

We claim that the elements of $C_{x_{0}}^{s, s^{\prime}}$, when $s^{\prime}<-s$, are "honest functions". In order to prove it, we shall suppose that $0<s<1, x_{0}=0$, and obtain that

$$
|f(x)-f(0)| \leq C|x|^{9} \text { when } 0<|x|<1 .
$$

Let $j_{0}$ be such that

$$
2^{-\left(j_{0}+1\right)}<|x| \leq 2^{-j_{0}}
$$

then

$$
\begin{aligned}
& |f(x)-f(0)| \leq \\
& \quad\left|S_{0} f(x)-S_{0} f(0)\right|+\sum_{0 \leq j \leq j_{0}}\left|u_{j}(x)-u_{j}(0)\right|+\sum_{j>j_{0}}\left|u_{j}(x)\right|+\sum_{j>j_{0}}\left|u_{j}(0)\right| .
\end{aligned}
$$

By definition,

$$
\left|u_{j}(x)\right| \leq C 2^{-j s}\left\{1+2^{j}|x|\right)^{-s^{\prime}} .
$$

Hence

$$
\left|\nabla u_{j}(x)\right| \leq C 2^{j(1-s)}\left(1+2^{j}|x|\right)^{-s^{2}} .
$$

So that, if $0 \leq j \leq j_{0}$,

$$
\left|u_{j}(x)-u_{j}(0)\right| \leq C_{1} 2^{j(1-s)}|x|
$$


and the total contribution of these terms is $C 2^{j(1-s)}$, which is equivalent to $C|x|^{\circ}$.

The serie $\sum_{j>j_{0}}|u j(x)|$ is bounded by $C \sum_{j>j_{0}} 2^{-j s}\left(2^{j}|x|\right)^{-s^{\prime}}$, which is equivalent to $C 2^{-j_{0} s}$, and the same estimate holds for $\sum_{j>j_{0}}\left|u_{j}(0)\right|$. Since, by Bernstein's inequality, $\left|S_{0} f(x)-S_{0} f(0)\right| \leq C_{0}|x|$, the result is proved. One can also easily check that, if $s>0$ and $s^{\prime}+s>0$, then $C_{x_{0}}^{s, s^{\prime}}$ is included in $C_{x_{0}}^{s}$.

In the next part, we shall examine the regularity of the elements of $C_{x_{0}}^{s,-s}$ at $x_{0}$.

\section{A comparison between $C_{x_{0}}^{s,-s}$ and $C_{x_{0}}^{s}$}

Let $s>0$, we saw that the elements of $C_{x_{0}}^{s,-s}$, cven restricted to $R^{n}-\left\{x_{0}\right\}$ are in general "wild distributions" for which (1) cannot hold. Though, we shall prove the following result.

Theorem 1. Let $s$ and $\beta$ be strictly positive numbers, and $u$ an element of $C_{x_{0}}^{s,-s} \cap C^{\beta}\left(\mathbf{R}^{n}\right)$. There exists a polynomial $P$ of degree less than s such that, if $\left|x-x_{0}\right| \leq 1$,

$$
|u(x)-P(x)| \leq C\left|x-x_{0}\right|^{s} \log \frac{2}{\left|x-x_{0}\right|},
$$

and this result is optimal.

Remark that, in this theorem, we arc looking for regular points in an irregular background, which is more subtle than the usual approach that consists in finding irregular points in a $C^{\infty}$ or analytical background (determination of the singular supports).

This theorem can be interpreted as a tauberian theorem. We have information on the behavior of averages of $f$ (its Littlewood-Paley decomposition) and a tauberian condition of minimal global regularity, which allow to obtain a pointwise result.

Proof of Theorem 1: Define $j_{0}$ and $j_{1}$ by

$$
2^{-j_{0}-1} \leq\left|x-x_{0}\right|<2^{-j_{0}} \text { and } j_{1}=\frac{s}{\beta} j_{0} .
$$

Let us restrict to the case $0<s \leq 1$ and $0<\beta<s$. Then $P(x)=u(0)$, and

$$
\begin{aligned}
|u(x)-u(0)| & \leq\left|S_{0} u(x)-S_{0} u(0)\right|+\sum_{0}^{j_{0}}\left|u_{j}(x)-u_{j}(0)\right|+ \\
& \sum_{j_{0}}^{j_{2}}\left(\left|u_{j}(x)\right|+\left|u_{j}(0)\right|\right)+\sum_{j_{1}}^{\infty}\left(\left|u_{j}(x)\right|+\left|u_{j}(0)\right|\right)=A+B+C+D .
\end{aligned}
$$


To estimate $A$ is a straightforward consequence of Bernstein's inequality. As concerns $B$, we use $u \in C_{0}^{s,-s}$, so that

$$
u_{j}(x)=2^{-j s} \beta_{j}\left(2^{j} x\right)
$$

where $\left|\beta_{j}(x)\right| \leq c(1+|x|)^{3}$. The Fourier transform of $\beta_{j}$ vanishes outside the set $1 / 2 \leq|\xi| \leq 2$, so that

$$
\left|\beta_{j}(x)-\beta_{j}(0)\right| \leq c|x| \text { if }|x| \leq 1
$$

hence

$$
\left|u_{j}(x)-u_{j}(0)\right| \leq c 2^{j}|x| 2^{-j s} .
$$

Adding up these inequalities, we get either $B \leq c|x|^{s}$ if $s<1$, or, if $s=1$, $B \leq c|x| j_{0} \leq c^{\prime}|x| \log \frac{2}{|x|}$.

In order to estimate $C$, remark that

$$
\left|u_{j}(x)\right| \leq c\left(2^{-j s}+|x|^{s}\right),
$$

so that $C$ is at most $O\left(|x|^{s}\left(j_{1}-j_{0}\right\}\right)=O\left(|x|^{s} \log \frac{2}{|x|}\right)$.

Because $u$ is in $C^{\beta},\left\|u_{j}\right\|_{\infty} \leq c 2^{-j \beta}$, so that $D$ is at most $O\left(2^{-j_{1} \beta}\right)=O\left(|x|^{s}\right)$, which ends the proof. The case $s>1$ is left to the reader.

One easily checks that, if (1) holds, then $u$ belongs to $C_{x_{0}}^{s,-9}$. So that, if $s+s^{\prime}>0$,

$$
C_{x_{0}}^{s, g^{\prime}} \subset C_{x_{0}}^{s} \subset C_{x_{0}}^{s,-s}
$$

The necessity to make the global $C^{\beta}$ asumption and the optimality of the logarithmic term in the result have been proved by Yves Meyer (personal communication), using wavelets and will be given in the next section.

\section{Wavelet coefficients and $C_{x_{0}^{*}}^{* y^{\prime}}$ spaces}

One of the interesting properties of the space $C_{x_{0}}^{s, s^{\prime}}$ is that it can be characterized by conditions on the wavelet coefficients. The intuitive reason for that is because the wavelet coefficients of a distribution arc given by a sampling (following Shannon's rule) on the filtering given by the Littlewood-Paley decomposition. It is therefore natural that spaces defined by local conditions on their Littlewood-Paley decomposition can be thus characterized. We assume in the following that the orthonormal basis of wavelets used has enough regularity and decay. We use the usual notations

$$
\psi_{j, k}(x)=2^{n j / 2} \psi\left(2^{j} x-k\right), j \in \mathbf{Z}, k \in \mathbf{Z}^{n} .
$$

Then, the following theorem is very easy to check. 
Theorem 2. A distribution u belongs to $C_{x_{0}}^{s, s^{t}}$ if and only if

$$
\left|<u, \psi_{j, k}>\right| \leq C 2^{-(n / 2+s) j}\left(1+\left|k-2^{j} x_{0}\right|\right)^{-s^{\prime}} .
$$

The other two-microlocal spaces can also be characterized by conditions on the wavelet coefficients. Recall that

$$
u \in H^{s, s^{\prime}} \Leftrightarrow\left\|2^{j s}\left(1+2^{j}|x|\right)^{s^{\prime}} u j\right\|_{L^{2} \leq c_{j}}
$$

with $\sum\left|c_{j}\right|^{2}<\infty$

Then, $u$ belongs to $H^{s, s^{\prime}}$ iff

$$
\sum 2^{j s}\left(1+2^{j}\left|\frac{k}{2^{j}}-x_{0}\right|\right)^{2 s^{\prime}}\left|C_{j, k}\right|^{2}<\infty
$$

We now give the counter-examples that show the optimality of Theorem 1 .

Assume that $\psi$ is a compactly supported wavelet, as constructed in [D]. One easily checks that it is possible to suppose

$$
\psi(0) \neq 0
$$

We first prove that the global $C^{\beta}$ asumption is needed in Theorem 1 .

Let $m$ be a positive integer and $\epsilon_{m}$ a real number such that $2^{m} \epsilon_{n n}$ is an integer, and $\epsilon_{m} \rightarrow 0$ when $m \rightarrow \infty$. The precise value of $\epsilon_{m}$ will be given later. Let $\alpha$ be such that $0<\alpha<1$. The wavelet coefficients of the counter-example $f$ are defined by:

if $2^{m} \leq j \leq 2^{m+1}$ and $k=\epsilon_{m} 2^{j}, C_{j, k}=2^{-j / 2} \epsilon_{m}^{\alpha}$;

else, $C_{j, k}=0$.

Then define

$$
f(x)=\sum_{m=0}^{\infty} f_{m}(x)
$$

with

$$
f_{m}(x)=\epsilon_{m}^{\alpha} \sum_{2^{m} \leq j \leq 2^{m+1}} \psi\left(2^{j}\left(x-\epsilon_{m}\right)\right)
$$

Choose then $\epsilon_{m}$ such that

$$
\frac{1}{2 m} \leq 2^{m} \epsilon_{m}^{\alpha} \leq \frac{1}{m}
$$

The supports of the $f_{m}$ are disjoint, $\left|f_{m}(x)\right| \leq C 2^{m} \epsilon_{m}^{\alpha}$, and $f(0)=0$. Then $f$ is continuous (because $2^{m} \epsilon_{m}^{\alpha} \rightarrow 0$ ). But

$$
f_{m}\left(\epsilon_{m}\right)=C 2^{m} \epsilon_{m}^{\alpha}
$$


so that

$$
\limsup \frac{\left|f(x)-f\left(x_{0}\right)\right|}{x^{\gamma}} \geq \limsup C 2^{m} \epsilon_{m}^{\alpha-\gamma}=+\infty \forall \gamma>0 .
$$

Hence $f$ is not in $C_{0}^{\gamma}$ for any value of $\gamma$, aithough condition (3) holds at 0 .

The following counter-example shows that the logarithmic term is needed in (6).

Take the same construction as before, but with

$$
\epsilon_{m}=2^{-\beta 2^{m}} \text { for a given } \beta>0 .
$$

Then

$$
\frac{\left|f\left(\epsilon_{m}\right)-f(0)\right|}{\epsilon_{m}^{\alpha}} \geq C 2^{m} \geq C^{\prime} \log \left|\epsilon_{m}\right| .
$$

Hence the optimality of the logarithmic term.

It should be noticed that other conditions similar to condition (7) can be introduced in order to be compared with other types of pointwise regularity conditions. For example, a comparison with pointwise differentiability is given by the following proposition, the proof of which is similar to the one of Theorem 1 .

Proposition 1. Let $f$ be a function differentiable at $x_{0}$ with wavelet coefficients $c_{j, k}$. Let $\lambda$ be the point $\left(k 2^{-j}, 2^{-j}\right)$ in the upper half-plane. Then, the following estimate holds

$$
\left|c_{j, k}\right| \leq C \eta(\lambda) 2^{-\left(\frac{n}{2}+1\right) j}\left(1+\left|k-2^{j} x_{0}\right|\right)
$$

where $\eta(\lambda) \leq 1$ and $\eta(\lambda)=o$ (1) when $\lambda$ tends to $\left(x_{0}, 0\right)$.

Conversely, if $f$ is in $C^{\beta}\left(\mathbf{R}^{n}\right)$ for a strictly positive $\beta$ and if there exists a positive function $\theta$ defined for positive values of $j$ such that $\sum \theta(j)<\infty$ and

$$
\left|c_{j, k}\right| \leq C \eta(\lambda) \theta(j) 2^{-\left(\frac{n}{2}+1\right) j}\left(1+\left|k-2^{j} x_{0}\right|\right),
$$

then $f$ is differentiable at $x_{0}$.

\section{Pseudo-differential operators and two-microlocalization}

We shall now study the action of generalized pseudo-differential operators on the spaces $C_{x_{0}}^{s, y^{\prime}}$. The operators $T$ that we shall consider will belong to the algebras $O p\left(M^{\gamma}\right)$ (cf. $[\mathrm{DJ}],[\mathrm{L}]$ and [M2]) defined by conditions on their distribution-kernel $K(x, y)$ as follows. 
Define $O^{\gamma}$ to be the class of operators such that, of the main diagonal, their distribution-kerncl $K$ is a function satisfying the following estimates: for any integer $\alpha$ such that $\alpha<\gamma$,

$$
\left|\partial^{\alpha} h(x, y)\right| \leq \frac{C}{|x-y|^{n+\alpha}} .
$$

If $\alpha$ is the integer such that $\gamma-1 \leq \alpha<\gamma$,

$$
\begin{aligned}
& \left|\partial^{\alpha} K(x, y)-\partial^{\alpha} K\left(x^{\prime}, y\right)\right| \leq \frac{C\left|x-x^{\prime}\right|^{\gamma-\alpha}}{|x-y|^{n+\gamma}} \text { if }\left|x-x^{\prime}\right| \leq \frac{|x-y|}{2}, \\
& \left|\partial^{\alpha} K(x, y)-\partial^{\alpha} K\left(x, y^{\prime}\right)\right| \leq \frac{C\left|y-y^{\prime}\right|^{\gamma-\alpha}}{|x-y|^{n+\gamma}} \text { if }\left|y-y^{\prime}\right| \leq \frac{|x-y|}{2},
\end{aligned}
$$

and the operator $T$ is such that $T\left(X^{\alpha}\right)=T^{*}\left(X^{\alpha}\right)=0$ for $\alpha$ less than or equal to $\gamma$.

The algebra $O p\left(M^{\gamma}\right)$ is then the union of all the $O^{\gamma^{\prime}}$ for $\gamma^{\prime}>\gamma$.

The usual pseudo-differential operators of order 0 are the sum of such an operator and of a regularizing operator.

Yves Meyer proved that the following caracterization holds (cf. [M2]).

Proposition 2. An operator $T$ belongs to $O p\left(M^{\gamma}\right)$ iff its "wavelet coefficients" defined by $c\left(\lambda, \lambda^{\prime}\right)=\left\langle T \psi_{\lambda}\right| \psi_{\lambda^{\prime}}>$ satisfy the following condition: there exists $\gamma^{\prime}>\gamma$, such that

$$
\left|c\left(\lambda, \lambda^{\prime}\right)\right| \leq \omega\left(\lambda, \lambda^{\prime}\right)
$$

with

$$
\omega\left(\lambda, \lambda^{\prime}\right)=C 2^{-\mid j-j^{\prime}\left(\left(\frac{n}{2}+\gamma^{\prime}\right)\right.}\left(\frac{2^{-j}+2^{-j^{\prime}}}{2^{-j}+2^{-j^{\prime}}+\left|\lambda-\lambda^{\prime}\right|}\right)^{n+\gamma^{\prime}}
$$

and

$$
\lambda=\left(k 2^{-j}, 2^{-j}\right)
$$

We shall now prove the following result.

Theorem 3. If $f$ belongs to $C_{x_{0}}^{s, s^{\prime}}$ and $T$ belongs to $O p\left(M^{\gamma}\right)$ with

$$
\gamma>\sup \left(\left|s+s^{\prime}\right|, s,\left|s^{\prime}\right|,-n-s\right),
$$

then $T(f)$ belongs to $C_{x_{0}}^{s, s^{\prime}}$.

If we keep Theorem 1 in mind, this theorem can be interpreted as follows. The position of the points of regularity of a function is essentially preserved under the action of singular integral operators such as the Hilbert transform. 
Proof of Theorem 3: Let $\theta(\lambda)=2^{-\left(\frac{n}{2}+s\right) j}\left(1+2^{j}\left|\lambda-x_{0}\right|\right)^{-s^{\prime}}$. We must prove that

$$
\sum_{\lambda} \omega\left(\lambda, \lambda^{\prime}\right) \theta(\lambda) \leq C \theta\left(\lambda^{\prime}\right)
$$

Notice that, for any $s^{t}$

$$
\left(1+2^{j}\left|\lambda-x_{0}\right|\right)^{-s^{\prime}} \leq\left(1+2^{j}\left|\lambda^{\prime}-x_{0}\right|\right)^{-s^{\prime}}\left(1+2^{j}\left|\lambda-\lambda^{\prime}\right|\right)^{\left|s^{\prime}\right|} .
$$

We split the sum $\sum \omega\left(\lambda, \lambda^{\prime}\right) \theta(\lambda)$ into two parts.

a) If $j \leq j^{\prime}$, then

$$
\begin{gathered}
\sum_{\lambda} \omega\left(\lambda, \lambda^{\prime}\right) \theta(\lambda) \leq C \sum_{\lambda} 2^{-\left(j^{\prime}-j\right)\left(\frac{n}{2}+\gamma\right)} \frac{2^{-\left(\frac{n}{2}+s\right) j}\left(1+2^{j}\left|\lambda-x_{0}\right|\right)^{-s^{\prime}}}{\left(1+2^{j}\left|\lambda-\lambda^{\prime}\right|\right)^{n+\gamma}} \\
\leq C \sum_{\lambda} 2^{-\left(j^{\prime}-j\right)\left(\frac{n}{2}+\gamma\right)} \frac{2^{-\left(\frac{n}{2}+s\right) j}\left(1+2^{j}\left|\lambda^{\prime}-x_{0}\right|\right)^{-s^{\prime}}}{\left(1+2^{j}\left|\lambda-\lambda^{\prime}\right|\right)^{n+\gamma-\left|s^{\prime}\right|}} \text { by }(8) \\
\leq C 2^{-\left(\frac{n}{2}+s\right) j^{\prime}} \sum_{\lambda} 2^{-\left(j^{\prime}-j\right)(\gamma-s)} \frac{\left(1+2^{j}\left|\lambda^{\prime}-x_{0}\right|\right)^{-s^{\prime}}}{\left(1+2^{j}\left|\lambda-\lambda^{\prime}\right|\right)^{n+\gamma-\left|s^{\prime}\right|}}
\end{gathered}
$$

We introduce now the two following subcases.

i) If $s^{\prime} \leq 0$ then

$$
\left(1+2^{j}\left|\lambda^{\prime}-x_{0}\right|\right)^{-s^{\prime}} \leq\left(1+2^{j^{\prime}}\left|\lambda^{\prime}-x_{0}\right|\right)^{-s^{\prime}}
$$

and

$$
\begin{gathered}
\sum_{\lambda} \omega\left(\lambda, \lambda^{\prime}\right) \theta(\lambda) \leq \theta\left(\lambda^{\prime}\right) \sum_{\lambda} \frac{2^{-\left(j^{\prime}-j\right)(\gamma-s)}}{\left(1+\left|k-2^{j} \lambda^{\prime}\right|\right)^{n+\gamma-\left|s^{\prime}\right|}} \\
\leq C \theta\left(\lambda^{\prime}\right) \text { if } \gamma>s \text { and } \gamma-\left|s^{\prime}\right|>0 .
\end{gathered}
$$

ii) If $s^{\prime}>0$ then

$$
\begin{gathered}
\left(1+2^{j}\left|\lambda^{\prime}-x_{0}\right|\right)^{-s^{\prime}}=2^{\left(j^{\prime}-j\right) s^{\prime}}\left(2^{\left(j^{\prime}-j\right)}+2^{j^{\prime}}\left|\lambda^{\prime}-x_{0}\right|\right)^{-s^{\prime}} \\
\leq 2^{\left(j^{\prime}-j\right) s^{\prime}}\left(1+2^{j^{\prime}}\left|\lambda^{\prime}-x_{0}\right|\right)^{-s^{\prime}}
\end{gathered}
$$

and

$$
\begin{gathered}
\sum_{\lambda} \omega\left(\lambda, \lambda^{\prime}\right) \theta(\lambda) \leq \theta\left(\lambda^{\prime}\right) \sum_{\lambda} \frac{2^{-\left(j^{\prime}-j\right)\left(\gamma-s-s^{\prime}\right)}}{\left(1+2^{j}\left|\lambda-\lambda^{\prime}\right|\right)^{n+\gamma-\left|s^{\prime}\right|}} \\
\leq C \theta\left(\lambda^{\prime}\right) \text { if } \gamma>s+s^{\prime} \text { and } \gamma-\left|s^{\prime}\right|>0 .
\end{gathered}
$$

b) If $j>j^{\prime}$, then 


$$
\sum_{\lambda} \omega\left(\lambda, \lambda^{\prime}\right) \theta(\lambda) \leq C \sum_{\lambda} 2^{-\left(j-j^{\prime}\right)\left(\frac{n}{2}+\gamma\right)} \frac{2^{-\left(\frac{n}{2}+s\right) j}\left(1+2^{j}\left|\lambda-x_{0}\right|\right)^{-s^{\prime}}}{\left(1+2^{j^{\prime}}\left|\lambda-\lambda^{\prime}\right|\right)^{n+\gamma}}
$$

We introduce again the two following subcases.

i) If $s^{\prime} \leq 0$ then

$$
\begin{gathered}
\sum_{\lambda} \omega\left(\lambda, \lambda^{\prime}\right) \theta(\lambda) \\
\leq C 2^{-\left(\frac{n}{2}+s\right) j^{\prime}} \sum_{\lambda} 2^{-\left(j-j^{\prime}\right)(n+\gamma+s)} \frac{\left(1+2^{j}\left|\lambda-x_{0}\right|\right)^{-s^{\prime}}}{\left(1+2^{j^{\prime}}\left|\dot{\lambda}-\lambda^{\prime}\right|\right)^{n+\gamma}}
\end{gathered}
$$

But $\left(1+2^{j}\left|\lambda-x_{0}\right|\right)^{-s^{\prime}} \leq 2^{-\left(j-j^{\prime}\right) s^{\prime}}\left(1+2^{j^{\prime}}\left|\lambda-x_{0}\right|\right)^{-s^{\prime}}$.

Hence

$$
\begin{aligned}
\sum_{\lambda} \omega\left(\lambda, \lambda^{\prime}\right) \theta(\lambda) \leq & C \theta\left(\lambda^{\prime}\right) \sum_{\lambda} \frac{2^{-\left(j-j^{\prime}\right)\left(n+\gamma+s+s^{\prime}\right)}}{\left(1+2^{j^{\prime}}\left|\lambda-\lambda^{\prime}\right|\right)^{n+\gamma-\left|s^{\prime}\right|}} \text { by }(8) \\
\leq & C \theta\left(\lambda^{\prime}\right) \sum_{j>j^{\prime}} 2^{-\left(j-j^{\prime}\right)\left(\gamma+s+s^{\prime}\right)} \\
& \leq C \theta\left(\lambda^{\prime}\right) \text { if } \gamma>-s-s^{\prime} .
\end{aligned}
$$

ii) If $s^{\prime}>0$ then

$$
\begin{gathered}
\sum_{\lambda} \omega\left(\lambda, \lambda^{\prime}\right) \theta(\lambda) \\
\leq C 2^{-\left(\frac{n}{2}+s\right) j^{\prime}} \sum_{\lambda} 2^{-\left(j-j^{\prime}\right)(n+\gamma+s)} \frac{\left(1+2^{j}\left|\lambda-x_{0}\right|\right)^{-s^{\prime}}}{\left(1+2^{j^{\prime}}\left|\lambda-\lambda^{\prime}\right|\right)^{n+\gamma}} \\
\leq C 2^{-\left(\frac{n}{2}+s\right) j^{\prime}} \sum_{\lambda} 2^{-\left(j-j^{\prime}\right)(n+\gamma+s)} \frac{\left(1+2^{j^{\prime}}\left|\lambda-x_{0}\right|\right)^{-s^{\prime}}}{\left(1+2^{j^{\prime}}\left|\lambda-\lambda^{\prime}\right|\right)^{n+\gamma}} \\
\leq C 2^{-\left(\frac{n}{2}+s\right) j^{\prime}} \sum_{\lambda} 2^{-\left(j-j^{\prime}\right)(n+\gamma+s)} \frac{\left(1+2^{j^{\prime}}\left|\lambda^{\prime}-x_{0}\right|\right)^{-s^{\prime}}}{\left(1+2^{j^{\prime}}\left|\lambda-\lambda^{\prime}\right|\right)^{n+\gamma-\left|s^{\prime}\right|}} \text { by (8) }
\end{gathered}
$$

$\leq C \theta\left(\lambda^{\prime}\right)$ as before.

Which ends the proof of Theorem 2. 


\section{Former pointwise regularity conditions and applications}

Some "good substitutes" for the $C_{x_{0}}^{s}$ condition were introduced already in 1961 by A.P.Calderón and A.Zygmund [CZ2]. They are called the $T_{u}^{p}$ classes.

A function $f$ belongs to $T_{u}^{p}\left(x_{0}\right)$ if there exists a polynomial $P$ of degree less than $u$ such that

$$
\left(\frac{1}{\rho^{n}} \int_{\left|x-x_{0}\right| \leq \rho}\left|f(x)-P\left(x-x_{0}\right)\right|^{p} d x\right)^{1 / p} \leq C \rho^{u},
$$

for $\rho$ small enough. Of course, if $f$ belongs to $C^{u}\left(x_{0}\right)$, then $f$ belongs to $T_{u}^{p}\left(x_{0}\right)$ for any $p$. This weak form of pointwise regularity is preserved under fractional integration and singular integral transformations. An application of these two properties is given by the following result. Since Calderon and Zygmund proved in a former paper ([CZ1]) that the inverse of an elliptic operator is the composition of a fractional integration and of a singular integral operator, they could deduce a theorem of pointwise regularity for elliptic operators in term of $T_{u}^{p}$ classes. A similar approach, has been followed by $\mathrm{E}$. Stein ([St]) for the definition of the harnonic derivative, which can be regarded as a condition on the behavior of the wavelet transform near the boundary of its definition domain.

However, the $T_{u}^{p}$ classes are not as closely related to the $C_{x_{0}}^{u}$ spaces as the $C_{z_{0}}^{g, s^{\prime}}$ are, and a resilt such as Theorem 1 cannot hold, as the following counterexample shows.

Let $f$ be an even function defined as follows:

$f$ is piecewise linear betwecn $2^{n}$ and $2^{n+1}$, for $n \in Z$

If $n$ is even, $f\left(2^{n}\right)=2^{c n}$

If $n$ is odd, $f\left(2^{n}\right)=0$.

Then $f$ belongs to $C^{\prime}(\mathbf{R})$, and, though it belongs to $T_{\frac{2}{p}+\epsilon^{\prime}}^{p}$ is not better than $C^{\epsilon}$ at 0 .

We shall now follow the ideas developped in $[\mathrm{CZ} 2]$ and get a pointwise regularity result for solutions of partial differential equations.

Theorem 4. Let $\Lambda$ be a partial differential operator of order $m$, with smooth cocfficients and elliptic at $x_{0}$. If $A f=g$ and $g$ belongs to $C_{x_{0}}^{s, s^{\prime}}$, then $f$ belongs to $C_{x_{0}}^{s+m, s^{\prime}}$.

The proof of this theorem makes use of the decomposition discovered by Calderon and Zygmund of the inverse of an elliptic operator as a product of a fractional integration and a pseudo-differential operator of order 0 . Such an operator is the sum of a regularizing operator and of an operator belonging to a class $O p\left(M^{\gamma}\right)$; so that Theorem 4 is an immediate consequence of the remarks of Part 1 and of Theorem 3. The following corollary gives an optimal result of pointwise Hölder regularity for elliptic operators and follows from Theorems 1 and 4 . 
Corollary 1. Let $\Lambda$ be a partial differential operator of order $m$, with smooth coefficients and elliptic at $x_{0}$. If $\Lambda f=g$, and if $g$ is a function that belongs to $C_{x_{0}}^{s}$, then there exists a polynomial $P$ of degree less than $s$ such that, for $\left|x-x_{0}\right| \leq 1$,

$$
|f(x)-P(x)| \leq C\left|x-x_{0}\right|^{3} \log \frac{2}{\left|x-x_{0}\right|} .
$$

This corollary uses the fact that, as Theorem 1 shows, the condition $C_{x_{0}}^{9,-s}$ is very closely related to $C_{x_{0}}^{s}$. Hence it could not be a consequence of the approach through the $T_{z}^{p}$ classes.

\section{The continuous wavelet transform}

It should be noticed that the characterizations that were given on the wavelet, coefficients hold not only in the case of an orthonormal basis of wavelets but also for the continuous wavelet transform. Let us recall at first the definition and some of the properties of this transform. Wc shall only consider the case of functions defined on $\mathbf{R}$. We need to suppose that the analysing wavelet $\psi$ is even or odd and has enough cancellation and decay.

For a strictly positive number $a$ and a real number $b$, define

$$
c(a, b)=\int \frac{1}{a} \psi\left(\frac{t-b}{a}\right) f(t) d t .
$$

Then $f$ can be recovered from its wavelet cocficients by

$$
f(t)=\int_{a>0} \frac{d a}{a^{2}} \int c(a, b) \psi\left(\frac{t-b}{a}\right) d b .
$$

Notice that we have taken a different normalization than in the orthonormal case.

This continuous transform secms more adapted to the study of "fractal type" functions because it is translation invariant and doesn't favour any particular scale. The same caracterizations as in the preceding parts can be proved for this transform. The proofs are exactly the same if one defines $u_{j}$ by

$$
u_{j}(t)=\int_{2^{-j}}^{2.2^{-j}} \frac{d a}{a^{2}} \int c(a, b) \psi\left(\frac{t-b}{a}\right) d b .
$$

Let us give the caracterization of $C_{z_{0}}^{s, s^{t}}$ as an example.

Proposition 3. A distribution belongs to $C_{x_{0}}^{s, s^{\prime}}$ if and only if its wavelet coefficients $c(a, b)$ satisfy the following estimate:

$$
|c(a, b)| \leq C a^{s+s^{\prime}} d\left((a, b),\left(0, x_{0}\right)\right)^{-s^{\prime}}
$$

where $d$ is the ustal euclidean distance.

Acknowledgement. The author expresses his thanks to $R$. Coifman and $Y$. Meyer for many enlightening discussions on the topics studied in this paper. 


\section{References}

[B1] J.M. BoNY, Interaction des singularités pour l'équation de Klein-Gordon non linéaire, Séminaire Goulaouic-Meyer-Schwartz 1983-84 Ecole Polytechnique.

[B2] 3.M. BONY, Second microlocalization and propagation of singularities for semi-linear hyperbolic equations, Taniguchi Symp. HERT. Katata (1984), 11-49.

[B3] J.M. BoNY, Calcul symbolique et propagation des singularités pour les équations aux dérivées partielles non linéaires, Ann.Scient.E.N.S. 14 (1981), 209-246.

[CZ1] A.P. CALDERón AND A. ZYGMund, Singular integral operators and differential equations, American J. Math. 79 (1957), 901-921.

[CZ2] A.P. CALDERÓN AND A. ZYGMUND, Local properties of solutions of elliptic partial differential equations, Studia Math. 20 (1961), 171-227.

[D] I. DAUBEchies, Orthonormal bases of compactly supported wavelets, Comm. on pure and Appl. Math. 41 (1988).

[DJ] G. DAVID AND J.L. JOURNÉ., A boundedness criterium for general Calderón-Zygmund operators, Ann. of Math. 120 (1984), 371-397.

[GKM] A. Grossmann, R. Kronland-Martinet and J. Morlet, Reading and understanding the continuous wavelet transform (preprint), C.P. T.7 Sec. 2 C.N.R.S. Luminy case 907 13288 MARSEILLE Cedex 9.

[H] M. HoLsCHNEIDER, On the wavelet transformation of fractal objects, $J$, Stat. Phys 50, 5-6 (1988).

[J1] S. JAFFARD, Exposants de Hölder en des points donnés et coefficients d'ondelettes, C.R.A.S. 308, Série 1 (1989), 79-81.

[J2] S. JaFfard, Thèse, Ecole Polytechnique (1989).

[J3] S. JAFFARD, "Construction of wavelets on open sets, A paraitre dans les Proceedings du Congrès Ondelettes et méthodes temps-fréquence," Springer-Verlag, Marseille, 1987.

[L] P.G. LEMARIÉ, Thèse de troisième cycle, Universitć d'Orsay, centre de Mathématiques.

[LM] P.G. LemariÉ ET Y. MEYer, Ondelettes et bases hilbertiennes, Revista Math. Beroamericana 1 (1986).

[Ma1] S. MALLAT, Multiresolution approximations and wavelet orthonormal bases of $L^{2}(R)$, Trans.A.M.S. (to appear).

[Ma2] S. MaLLaT, A theory for Multiresolution signal decomposition: The wavelet representation, I.E.E.E. on pattern analysis and machine intelligence (to appear).

[M1] Y. MEYER, "Principe d'incertitude, bases hilbertiennes et algèbres d'opérateurs," Séminaire Bourbaki 38 ${ }^{e}$ année 85-86 no. 662. 
[M2] Y. MEYER, "Ondelettes et opérateurs," Hermann, 1990.

[M3] Y. MEYER, Ondelettes, fonctions splines et analyses graduées (Cahiers de Mathématiques de la décision, CEREMADE, Université Paris-Dauphine, 75775 Paris Cedex 16).

[M4] Y. MEYER, "Wavelets and aperators," (idern).

[St] E.STEIN, "Singular integrals and differentiability properties of functions," Princeton University Press, 1970.

[S] J.O. STROMBERG, "A modified Franklin system and higher order spline systems on $R^{n}$ as unconditionnal bases for Hardy spaces," Conference in honor of Antoni Zygmund 2, W. Beckner ed. Wadsworth Math series, pp. 475-493.

C.E.R.M.A.

Ecole Nationale des Ponts et Chazussies

La Courtine, 93167 Noisy-le-grand

FRANCE 